\title{
Molecular Gas Rich Galaxy Pair Unveiled in the Lensed Quasar $0957+561$
}

\author{
P. Planesas, J. Martin-Pintado
}

Observatorio Astronómico Nacional (IGN), Apartado 1143, E-28800 Alcalá de Henares (Spain)

R. Neri

Institut de Radio Astronomie Millimetrique (IRAM), F-38469 Saint Martin d'Heres (France)

L. Colina

Instituto de Fisica de Cantabria (CSIC-UC), Facultad de Ciencias, E-39005 Santander (Spain)

\begin{abstract}
Molecular gas in the host galaxy of the lensed quasar QSO $0957+561$ has been detected at the redshift of 1.414 using the IRAM Plateau de Bure interferometer. This detection shows the extended nature of the molecular gas distribution. The molecular gas mass is $2-410^{9}$ $\mathrm{M}_{\odot}$ and it is distributed in a disk that is several kiloparsecs in diameter. A second, weaker component of $\mathrm{CO}$ is interpreted as arising from a close companion galaxy moving at a velocity $440 \mathrm{~km} \mathrm{~s}^{-1}$ higher than the host galaxy.
\end{abstract}

\section{Introduction}

Gravitational lenses have recently become a powerful tool for probing the molecular gas content in galaxies at high redshift $(z>1)$. In most cases, magnification of the emitted spectral lines by a gravitational lens has helped to make the molecular gas emission detectable. However, prior to our observations, the emission had not been resolved in any high redshift object.

In 1998 we observed the $3.1 \mathrm{~mm}$ radio continuum and the CO 2-1 emission of QSO $0957+561$ using the radio interferometer of the Institute de RadioAstronomie Millimetrique (IRAM) located at Plateau de Bure (France). The synthesized beam was $3.2^{\prime \prime} \times 3.1^{\prime \prime}$. Radio continuum emission was detected towards the $\mathrm{A}$ and $\mathrm{B}$ quasar images and the western radio jet.

Our detection of the CO 2-1 emission of the QSO $0957+561$ host galaxy shows that radio spectroscopy of molecular lines with high-angular and highvelocity resolution of lensed galaxies at cosmological distances is able to trace the extended distribution of the molecular gas, even if it is unresolved by the highest angular resolution currently available, and its kinematics. 


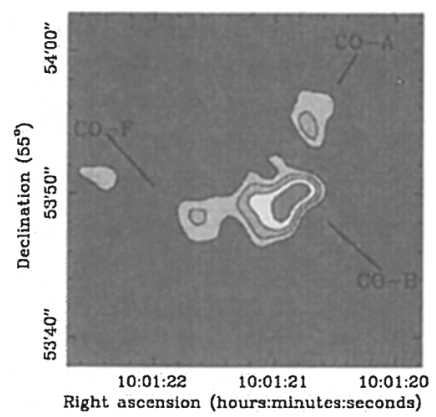

Figure 1. Contour map of the integrated intensity of the CO 2-1 emission. The contour levels are $0.30,0.43,0.57$ and $0.70 \mathrm{Jy} \mathrm{km} \mathrm{s}{ }^{-1}$.

\section{Results and Discussion}

Two images, labelled CO-A and CO-B in Figure 1, lay close to the radio continuum and optical A and B images. The CO-B image is a highly distorted arc, extended in the east-west direction. A third image of the molecular gas emission, CO-F, with no counterpart in the radio continuum or optical wavelengths, is connected to the CO-B image by a weak arclike structure. The resulting overall picture is interpreted as produced by the lens acting on an extended distribution of the molecular gas in the host galaxy. We found a lower limit of $2^{\prime \prime}$ $(12 \mathrm{kpc})$ for the angular size of the gas distribution in the quasar host galaxy.

The numerical results that follow, previously published by Planesas et al. (1999), have been corrected by a factor $1 / 1.5$ in the velocity scale, due to an improper treatment of the file headers by the reduction package.

The line profile taken toward the CO-A image shows two velocity components. The blue component peaks at about the same velocity as CO-B. The red component peaks at a velocity $440 \mathrm{~km} \mathrm{~s}^{-1}$ higher than the blue one, and it is interpreted as arising from a second object, a molecular gas rich galaxy that has not been detected in previous observations. The companion galaxy to the quasar host galaxy would be located a few tenths of an arc second towards the north of the quasar, above the outer northern caustic of the lens, as the radio jets are.

The molecular gas masses estimated from the emission toward the quasar host galaxy observed in images A and B are 2 and $4 \times 10^{9}$ solar masses, respectively, for a luminosity distance of $6.9 \mathrm{Gpc}$ and a magnification factor of $m \sim 10$ for every component. This mass is similar to the molecular gas mass of the Milky Way and is distributed in a disk that is several kiloparsecs in diameter. The molecular gas mass of the companion galaxy is $10^{9}$ solar masses. These masses are one order of magnitude smaller than the masses typically found in the host galaxies of quasars at $z>1$.

\section{References}

Planesas, P., Martin-Pintado, J., Neri R., \& Colina, L. 1999, Science 286, 2493 